\title{
A FLUIDELASTIC MODEL FOR THE NONLINEAR DYNAMICS OF TWO-DIMENSIONAL INVERTED FLAGS
}

\author{
Mohammad Tavallaeinejad \\ Michael Païdoussis, Mathias Legrand \\ Department of Mechanical Engineering \\ McGill University \\ Montréal, Québec, H3A 0C3 \\ Canada
}

\author{
Mojtaba Kheiri \\ Department of Mechanical, \\ Industrial and Aerospace Engineering \\ Concordia University \\ Montréal, Québec, H3G 1 M8 \\ Canada
}

\begin{abstract}
A nonlinear fluid-elastic model is proposed for the study of the dynamics of inverted flags. The quasi-steady version of Theodorsen's unsteady aerodynamic theory is used for inviscid fluid-dynamic modelling of the deforming flag in axial flow. Polhamus's leading edge suction analogy is employed to model flow separation effects from the free end at moderate angles of attack via a nonlinear vortex-lift force. The flag is modelled structurally via a geometrically-exact Euler-Bernoulli beam theory. Using the extended Hamilton's principle, the nonlinear partial-integrodifferential equation governing the dynamics of the inverted flag in terms of the angle of rotation of the flag is obtained. The equation of motion is discretised spatially via the Galerkin method and is integrated in time via Gear's backward differentiation formula. The bifurcation diagrams are obtained using a time-integration method and pseudo-arclength continuation. It is shown that inverted flags undergo multiple bifurcations with respect to flow velocity, and they generally exhibit four dynamical states: (i) stretched-straight, (ii) buckled, (iii) deflected-flapping, and (iv) large-amplitude flapping. Also, flapping of inverted flags probably develops through fluid-elastic instabilities. Our findings suggest that the system dynamics is sensitive to the mass ratio. It is shown that the mass ratio parameter does not affect the stability of the stretched-straight state and the onset of divergence; however, it controls the possibility of a direct transition from static undeflected equilibrium to large-amplitude flapping motion and it affects the amplitude of large-amplitude flapping.
\end{abstract}

\section{NOMENCLATURE}

$\begin{array}{ll}L, h & \text { Flag length, thickness } \\ \rho_{\mathrm{p}}, \rho_{\mathrm{f}} & \text { Flag, fluid mass density } \\ v & \text { Poisson ratio } \\ D & \text { Flag flexural rigidity } \\ \eta & \text { Material viscosity coefficient } \\ U & \text { Free stream fluid flow velocity } \\ R e & \text { Reynolds number } \\ \Pi & \text { Dimensionless flow velocity } \\ \mu & \text { Mass ratio } \\ f, T & \text { Frequency, period of oscillation } \\ f_{\mathrm{R}} & \text { Reduced frequency } \\ \omega & \text { Complex eigenfrequencies }\end{array}$

$u(x, t) \quad$ Longitudinal in-plane deflection

$w(x, t) \quad$ Transverse deflection

$\psi(x, t) \quad$ Cross-section rotation angle

$\kappa(x, t) \quad$ Curvature of the mid-plane

$e(x, t) \quad$ Strain of the mid-plane

$\mathcal{T}(t) \quad$ Flag kinetic energy

$\mathcal{V}(t) \quad$ Flag potential energy

$\mathcal{W}_{\mathrm{f}}(t) \quad$ Virtual work of fluid forces

$\mathcal{W}_{\mathrm{d}}(t) \quad$ Virtual work of damping

$V_{\mathrm{n}}(x, t) \quad$ Normal component of relative velocity in oxz

$V_{\tau}(x, t) \quad$ Tangential component of relative velocity in oxz

$q_{i}(t) \quad i$ th generalized coordinate

$\Psi_{i}(x) \quad i$ th eigenfunction

OXZ Undeformed, inertial, Cartesian coordinate

oxz Deformed local, orthogonal curvilinear coordinate

OXX $\bar{Z} \quad$ Cartesian coordinate system in the conformally mapped plane

$\operatorname{or} \theta \quad$ Polar coordinate system in the conformally mapped plane

$q_{r}(\theta, t) \quad$ Radial component of the velocity in $r, \theta$ coordinate system

$q_{\theta}(\theta, t) \quad$ Tangential component of the velocity in $r, \theta$ coordinate system

$P_{\text {nc }}(\theta, t) \quad$ Non-circulatory component of the pressure

$P_{\mathrm{c}}(\theta, t) \quad$ Circulatory component of the pressure

$F_{\mathrm{p}}(\theta, t) \quad$ Nonlinear vortex force

$F_{\mathrm{N}}(\theta, t) \quad$ Total normal force acting on the flag

$\partial_{t} \bullet, \bullet \quad$ First time derivative

$\partial_{t t} \bullet, \ddot{\bullet} \quad$ Second time derivative

$\partial_{x} \bullet, \bullet x \quad$ First space derivative

$\partial_{x x} \bullet \quad$ Second space derivative

-* Dimensionless counterpart of $\bullet$

$\delta \bullet \quad$ Variation of $\bullet$ in Hamilton's Principle

\section{INTRODUCTION}

The flapping of inverted flags (that is a thin free-clamped plate, free at the upstream end and clamped at the downstream one) was first investigated theoretically by Guo and Païdoussis [1], who studied the linear stability of rectangular plates with various boundary conditions in inviscid, incompressible two-dimensional (2-D) flow. They found that a free-clamped (or inverted) plate 
flutters at a non-zero critical velocity inversely proportional to the fluid-to-plate mass ratio. Recently, interest in the dynamics of inverted flags has been revived mainly due to their potential application to small-scale energy harvesting: see, for example, the experimental study on inverted piezoelectric flags by Orrego et al. [2]. Moreover, exploring the dynamics of inverted flags will improve our understanding of the flapping of biological structures, such as leaves in wind [3,4].

Kim et al. [5] performed the first experiments on inverted flags in wind and water tunnels. They identified three consecutive regimes/modes of dynamical behaviour, namely the straight mode, the flapping mode and the fully deflected mode, as the flow velocity in the test-section was increased. It was found that the inverted flag performed flapping only within a finite range of flow velocity, somewhat analogously to vortex-induced vibration (VIV) of a spring-supported cylinder in cross-flow. It was also found that the onset of instability (i.e. divergence and initiation of the largeamplitude flapping) is relatively insensitive to the mass ratio.

In an elaborate study, Sader et al. [3] employed a combination of a mathematical approach based on the steady-state aerodynamic theory by Kornecki et al. [6], a scaling analysis and experimental measurements to understand the underlying physical mechanism of inverted flag flapping. They concluded that the large-amplitude flapping motion of an inverted flag is a vortex-induced oscillation, in contrast to flapping of a conventional flag, which is a 'selfexcited' vibration. However, they concluded that classical VIV does not occur for heavy flags (i.e. small mass ratios). Moreover, they showed that the onset of flapping is due to a divergence instability, and thus, is independent of the mass ratio.

In a subsequent paper, Sader et al. [7] examined theoretically the stability of zero-aspect-ratio flexible and flexibly-supported rigid inverted flags in a uniform steady axial flow. They found that, at low flow velocities, the zero-deflection equilibrium is the only possible stable state; however, at a critical flow velocity, a saddle-node bifurcation occurs, from which a new deflected equilibrium state emanates and grows in amplitude as the flow velocity is increased. Their experimental measurements for low aspect ratio inverted flags agree well with their theoretical results both qualitatively and quantitatively.

This work was pursued by Tavallaeinejad et al. [8,9] who developed nonlinear theoretical models to explore the static and dynamic response of flexible low aspect ratio inverted flags in axial flow. They found that a low aspect ratio flag undergoes a static divergence via a subcritical pitchfork bifurcation followed by a saddle-node bifurcation. However, beyond a critical aspect ratio, relatively heavy (i.e. $\mu \leq 0.4$ ) inverted flags go through the following sequence of dynamical states, as the flow velocity is increased: (i) stable at the rest position, (ii) flapping about the rest position via a supercritical Hopf bifurcation (i.e. symmetric flapping mode), (iii) flapping about a deflected equilibrium (i.e. asymmetric flapping or deflected-flapping mode) via two saddlenode bifurcations, and (iv) highly deflected shape on one side via another Hopf bifurcation. Increasing the mass ratio (from $\mu=0.1$ to $\mu=0.4$ ) was found to lower the critical flow velocity for flapping and to increase the flapping amplitude. The latter differs from the experimental observations reported in [5].

In the present study, an analytical nonlinear model is developed to investigate the dynamics and post-critical behaviour of inverted flags in axial flow. In particular, we focus on the dynamics of high aspect-ratio heavy inverted flags by making use of inviscid flow aerodynamic theory. Thus, a continuum representation of flow forces is detailed based on the quasi-steady version of 2-D unsteady aerodynamic theory [10] to formulate the reactive force and Polhamus's leading-edge suction analogy [11] to deal with the separated flow at the flag leading edge. The resulting equation of motion is solved numerically to obtain the onset of instabilities together with the frequency and amplitude of flapping.

\section{ANALYTICAL MODELLING}

The system of interest is shown schematically in Fig. 1. It consists of a vertical cantilevered thin plate subjected to an inviscid axial flow impinging on its free end, with mean flow velocity $U$. Two assumptions are made here: (i) the spanwise deformation of the flag is neglected, and (ii) the flag is assumed to be infinitely wide (i.e. $R \rightarrow \infty$ ). Accordingly, the aerodynamic forces acting on the flag may be approximated by a 2-D incompressible flow theory.

Two coordinate systems are adopted: (i) a right-handed rectangular Cartesian reference system OXZ, with the $X\left(\mathbf{e}_{X}\right)$ and $Z\left(\mathbf{e}_{Z}\right)$ axes being in the axial and transverse direction, respectively, (ii) a curvilinear coordinate system oxz, with the $\mathrm{x}$-axis being along the flag from its clamped end towards the free one. The unit vectors $\mathbf{e}_{x}$ and $\mathbf{e}_{z}$ are tangential and normal to the centreline, respectively.

Taking into account the inextensibility assumption, all physical quantities can be expressed in in terms of $(x, t)$ (that is the coordinate system embedded in the flag). Moreover, the rotation angle $\psi$ and the curvature $\kappa^{1}$ are related to the longitudinal, $u$, and transverse, $w$, motions of a generic point at a distance $z$ from the mid-plane on the cross-section by $\sin \psi=\partial_{x} w, \cos \psi=1+\partial_{x} u$, and $\kappa=\partial_{x} \psi$, where $\partial_{x}$ denotes the first spatial derivative. This condition reduces the number of dependent variables to one, and $w$ and $u$ can be expressed in terms of the rotation angle of the crosssection $\psi$, which now becomes the primary variable for describing the flag motion. Derivation of the equation of motion in terms of $\psi$ allows for the prediction of large-amplitude deformations even when the tip rotation exceeds $\pi / 2$ [9].

The kinetic and potential energies of the inextensible inverted flag in terms of the rotation angle read

$$
\begin{aligned}
\mathcal{T} & =\frac{1}{2} \rho_{\mathrm{p}} h \int_{0}^{L}\left(\left[\int_{0}^{x} \sin \psi(s, t) \dot{\psi}(s, t) \mathrm{d} s\right]^{2}\right. \\
& \left.+\left[\int_{0}^{x} \cos \psi(s, t) \dot{\psi}(s, t) \mathrm{d} s\right]^{2}\right) \mathrm{d} x+\frac{1}{24} \rho_{\mathrm{p}} h^{3} \int_{0}^{L} \dot{\psi}^{2} \mathrm{~d} x
\end{aligned}
$$

and

$$
\mathcal{V}=\frac{1}{2} D \int_{0}^{L} \psi_{x}^{2} \mathrm{~d} x
$$

in which $D=E h^{3} /\left(12\left(1-v^{2}\right)\right)$ is the plane-strain flexural rigidity for plates of large aspect ratio. The virtual work done

\footnotetext{
${ }^{1}$ In the remainder, when space and time dependencies in the various quantities used in the derivations are obvious, they are omitted for conciseness purposes. The uncertain reader is referred to the nomenclature.
} 


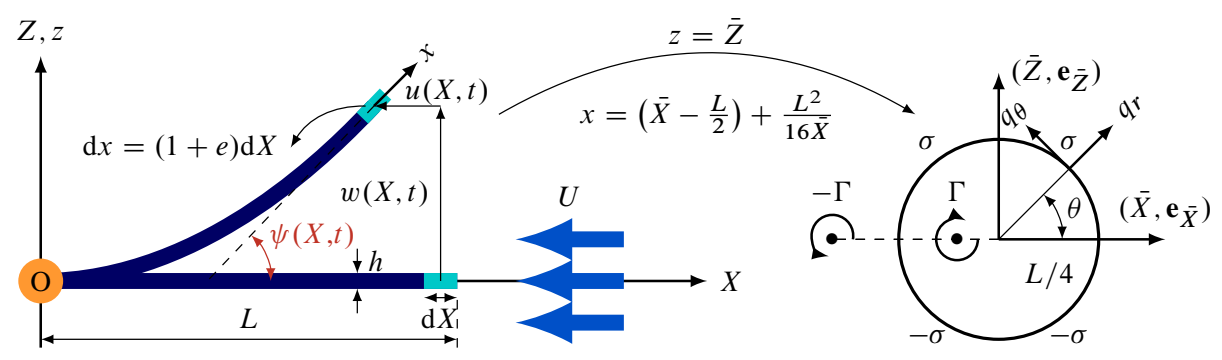

FIGURE 1: (Left) Flag idealised as an inextensible cantilevered beam. An arbitrary infinitesimal element $\mathrm{d} X$ of the beam undergoes longitudinal $u$ and transverse $w$ displacements dependent to each other by the rotation angle $\psi$. (Right) Joukowski's conformal mapping between the plate (i.e. XZ-plane) and the circle of radius $L / 4$ (i.e. $\bar{X} \bar{Z}$-plane); $q_{r}$ and $q_{\theta}$ are the radial and circumferential velocities on the circle in the $\bar{X} \bar{Z}$-plane, respectively. The impermeability condition is satisfied by considering a set of sources and sinks of strength $\sigma$ on the top and bottom halves of the circle, respectively. The induced velocity due to a single bound vortex of strength $\Gamma$ and its image (i.e. the wake vortex of strength $-\Gamma$ ) at an arbitrary point on the circle satisfies the Kutta condition.

by the Kelvin-Voigt type of internal dissipation and by the fluidrelated force normal to the flag may be written as

$$
\begin{aligned}
& \delta \mathcal{W}_{\mathrm{d}}=-D \eta \int_{0}^{L} \partial_{x x} \dot{\psi} \delta \psi \mathrm{d} x, \\
& \delta \mathcal{W}_{\mathrm{F}}=\int_{0}^{L} F_{\mathrm{N}}(\cos \psi \delta w-\sin \psi \delta u) \mathrm{d} x,
\end{aligned}
$$

where $\eta$ stands for the viscoelastic damping coefficient, and $F_{\mathrm{N}}$ denotes the normal force acting on the flag, which is now derived.

The relative velocity between the solid (or body) and the incident flow in the inertial frame reads as: $\mathbf{V}_{\text {rel }}=(\dot{u}+U) \mathbf{e}_{x}+\dot{w} \mathbf{e}_{z}$. Upon projecting $\mathbf{V}_{\text {rel }}$ onto the tangential and normal directions, the velocity of an element of the body with respect to the flow, expressed in the deformed coordinate system may be written as $\mathbf{V}_{\text {rel }}=V_{\tau} \mathbf{e}_{X}+V_{\mathrm{n}} \mathbf{e}_{Z}$, where

$$
\begin{aligned}
& V_{\tau}=(\dot{u}+U) \cos \psi+\dot{w} \sin \psi, \\
& V_{\mathrm{n}}=-(\dot{u}+U) \sin \psi+\dot{w} \cos \psi,
\end{aligned}
$$

in which

$$
\begin{aligned}
& \dot{u}=-\int_{0}^{x} \dot{\psi}(s, t) \sin \psi(s, t) \mathrm{d} s, \\
& \dot{w}=\int_{0}^{x} \dot{\psi}(s, t) \cos \psi(s, t) \mathrm{d} s .
\end{aligned}
$$

The pressure-related forces corresponding to the motion of the inverted flag in an inviscid fluid flow are modelled based on 2D quasi-steady thin airfoil theory involving large angles of attack $[12,13,14]$. First, the quasi-steady forces are derived using a velocity potential approach in a way that the solution is divided into non-circulatory and circulatory contributions, each part of which is obtained most conviniently using Joukowski's conformal transformation (see Fig. 1). Next, the leading edge separated flow effects are modelled utilizing Polhamus's leading-edge suction analogy [11].

The pressure distribution over the flag due to the noncirculatory contribution of the flow in the $\overline{\mathrm{X}} \overline{\mathrm{Z}}$ plane at $r=L / 4$ may be written as

$$
\begin{gathered}
\Delta P^{\mathrm{nc}}(\theta, t)=-\rho\left(\frac{L}{2} \partial_{t}\left(\int_{\theta}^{0} \int_{0}^{\pi} \frac{V_{\mathrm{n}}(\varphi, t) \sin ^{2} \varphi}{\cos \varphi-\cos \theta} \mathrm{d} \varphi \mathrm{d} \theta\right)\right. \\
\left.-\frac{(\dot{u}+U) \cos \psi+\dot{w} \sin \psi}{\sin \theta} \int_{0}^{\pi} \frac{V_{\mathrm{n}}(\varphi, t) \sin ^{2} \varphi}{\cos \varphi-\cos \theta} \mathrm{d} \varphi\right),
\end{gathered}
$$

noting that $x=(1+\cos \theta) / 2$. Applying the Kutta condition at the trailing edge (i.e. $\theta=\pi)^{2}$ and the quasi-steady assumption, ${ }^{3}$ which neglects only the influence of the shed vortices on the flow field [12], the pressure distribution originating from the circulatory contribution of the flow may be written as

$$
\Delta P^{\mathrm{c}}(\theta, t)=-\frac{\rho V_{\tau}}{\sin \theta} \frac{2}{\pi} \int_{0}^{\pi} V_{\mathrm{n}}(\varphi, t)(1-\cos \varphi) \mathrm{d} \varphi .
$$

The nonlinear vortex lift associated to the leading edge vortex may be expressed in terms of the quasi-steady force obtained in Eq. (6). Integration of the pressure around a contour at the leading edge, and application of Bernoulli's equation yields

$$
F_{\mathrm{p}}=\frac{\rho_{\mathrm{f}} L}{16 \pi} \int_{0}^{\pi} V_{\mathrm{d}}(\varphi, t) \mathrm{d} \varphi\left|\int_{0}^{\pi} V_{\mathrm{d}}(\varphi, t) \mathrm{d} \varphi\right|,
$$

in which $V_{\mathrm{d}}(\varphi, t)=V_{\mathrm{n}}(\varphi, t)(1+\cos \varphi)$, and the absolute operator is used to modify the force to be always aligned with motion.

Hence, the total quasi-steady normal force acting on the inverted flag is

$$
F_{\mathrm{N}}(\theta, t)=-\left(\Delta P^{\mathrm{nc}}+\Delta P^{\mathrm{c}}\right)-2 F_{\mathrm{p}}(t) \delta_{\mathrm{D}}(\cos \theta-1),
$$

\footnotetext{
${ }^{2}$ The validity of the Kutta condition for unsteady flows is a challenging issue in the theoretical aerodynamics. Previous studies on oscillating airfoils in flow, e.g. $[15,16]$, suggest that for oscillations of low frequency, small amplitudes and small angles of attack, the Kutta condition may still be applied to the trailing edge of the airfoil. Thus, for an inverted flag which is free at the leading edge and clamped at the trailing edge, it may appear reasonable to apply the Kutta condition at the trailing edge. The reader is also referred to Ref. [17] for more details.

${ }^{3}$ This is an approximation of Theodorsen theory, in which $C(k) \rightarrow 1$, with $C(k)$ being the Theodorsen function based on Hankel functions of the second kind, and $k$ denoting the reduced frequency. The quasi-steady theory can be used in the time domain for slow harmonic oscillations with small $k$, or slowly varying motion that is not harmonic [18].
} 
with $\delta_{\mathrm{D}}$ denoting the Dirac delta function. Note that the end-shear due to the force that results from Polhamus' effect at the leading edge is transferred from the boundary condition into the equation of motion.

Inserting (9) into (3) yields the virtual work done by the fluidrelated forces normal to the flag. Substituting the resultant together with Eqs. (1) to (2) into the generalised Hamilton's principle and performing several manipulations, as well as utilizing the dimensionless parameters $x^{*}=x / L, t^{*}=t /\left(\sqrt{\rho_{\mathrm{p}} h / D} L^{2}\right), \beta=$ $h^{2} /\left(12 L^{2}\right), \eta_{\mathrm{d}}=\eta / \tau, \mu=\rho_{\mathrm{f}} L /\left(h \rho_{\mathrm{p}}\right)$ and $\Pi=L U \sqrt{\rho_{\mathrm{f}} L / D}$, one obtains the following nonlinear dimensionless equation of motion solely expressed in terms of the rotation angle $\psi$ :

$$
\begin{aligned}
& \beta \ddot{\psi}-\partial_{x x} \psi-\eta_{\mathrm{d}} \partial_{x x} \dot{\psi} \\
& -\sin \psi \partial_{t t}\left(\int_{x}^{1} \int_{0}^{s} \cos \psi(\xi, t) \mathrm{d} \xi \mathrm{d} s\right) \\
& +\cos \psi \partial_{t t}\left(\int_{x}^{1} \int_{0}^{s} \sin \psi(\xi, t) \mathrm{d} \xi \mathrm{d} s\right) \\
& +\cos \psi \int_{1}^{x} F_{\mathrm{N}}^{*}(s, t) \cos \psi(s, t) \mathrm{d} s \\
& +\sin \psi \int_{1}^{x} F_{\mathrm{N}}^{*}(s, t) \sin \psi(s, t) \mathrm{d} s=0,
\end{aligned}
$$

where

$$
\begin{aligned}
& F_{\mathrm{N}}^{*}=\frac{\mu}{\pi} \int_{\theta}^{0} \int_{0}^{\pi}[(\ddot{w}-\dot{u} \dot{\psi}) \cos \psi-(\ddot{u}+\dot{w} \dot{\psi}) \sin \psi] f \mathrm{~d} \varphi \mathrm{d} \vartheta \\
& -\frac{\Pi \sqrt{\mu}}{\pi} \int_{\theta}^{0} \int_{0}^{\pi} \dot{\psi} \cos \psi f \mathrm{~d} \varphi \mathrm{d} \vartheta \\
& -\frac{2}{\pi \sin \theta}\left[\mu(\dot{u} \cos \psi+\dot{w} \sin \psi) \int_{0}^{\pi}(\dot{w} \cos \psi-\dot{u} \sin \psi) g \mathrm{~d} \varphi\right. \\
& -\Pi \sqrt{\mu}(\dot{u} \cos \psi+\dot{w} \sin \psi) \int_{0}^{\pi} \sin \psi g \mathrm{~d} \varphi \\
& \quad+\Pi \sqrt{\mu} \cos \psi \int_{0}^{\pi}(\dot{w} \cos \psi-\dot{u} \sin \psi) g \mathrm{~d} \varphi \\
& \left.\quad-\Pi^{2} \cos \psi \int_{0}^{\pi} \sin \psi g \mathrm{~d} \varphi\right] \\
& -\frac{1}{16 \pi} \int_{0}^{\pi}(1+\cos \varphi)(\Pi \sqrt{\mu} \dot{w} \cos \psi-(\Pi \sqrt{\mu} \dot{u}+1) \sin \psi) \mathrm{d} \varphi \\
& \times\left|\int_{0}^{\pi}(1+\cos \varphi)(\Pi \sqrt{\mu} \dot{w} \cos \psi-(\Pi \sqrt{\mu} \dot{u}+1) \sin \psi) \mathrm{d} \varphi\right| \delta_{\mathrm{D}}(s-1),
\end{aligned}
$$

in which $g \equiv g(\varphi, \theta), f \equiv f(\varphi, \vartheta), \psi \equiv \psi(\xi, t), w \equiv w(\xi, t)$, $u \equiv u(\xi, t), \xi=(1+\cos \varphi) / 2, f(\varphi, \vartheta)=\sin ^{2} \varphi /(\cos \varphi-$ $\cos \theta)$, and $g(\varphi, \theta)=(1-\cos \varphi)(1+\cos \theta) /(\cos \varphi-\cos \theta)$. The clamped-free boundary conditions are $\psi(0, t)=0$ and $\psi_{x}(L, t)=0$. Note that the asterisk notation of $x^{*}$ and $t^{*}$ has been dropped throughout for simplicity.

The singular integral in Eq. (6) is evaluated using Glauert's principal value integral [16]. To this end, the normal component of the relative velocity is expressed in a succession Taylor's expansions. This results in a polynomial representation of the source/sink sheet strength in terms of $\psi$. In this paper, third order Taylor's series expansions are retained.
The spatial discretisation of the nonlinear equation of motion given in (10) is made by employing the Galerkin method. Thus, we may write

$$
\psi(x, t)=\sum_{i=1}^{M} \Psi_{i}(x) q_{i}(t),
$$

where $\Psi_{i}(x)$ represent suitable comparison functions and $q_{i}(t)$ are the corresponding unknown time-dependent generalized coordinates; $M$ denotes the number of modes utilized. Applying Galerkin's technique results in a set of $M$ second-order ordinary differential equations (ODEs) with trigonometric terms. Integrals may not be evaluated in closed form due to the presence of trigonometric terms as well as the absolute function. Hence, integrations in space are computed numerically by evaluating the integrand at equally spaced points over the domain, retaining sufficient number of terms to ensure converged results.

In this study, six generalized coordinates are retained in the series expansion of the rotation angle, resulting in six secondorder ODEs. These equations are then recast into state-space form, resulting in a set of 12 first-order ODEs. These equations are then solved using direct time integration via Gear's backward differentiation formula (BDF) [19], suitable for the problem at hand, yielding the time-varying generalised coordinates $q_{i}(\tau)$. In order to construct the bifurcation diagrams, the stable periodic solutions are numerically traced via direct time integration using the calculated generalised coordinates for a given flow velocity as initial conditions for the next flow velocity.

For linear dynamics, a standard eigenvalue problem is solved to determine the eigenfrequencies of the system and assess the stability.

\section{NUMERICAL RESULTS}

In this section, linear aspects of the dynamics, such as the mechanism of instability and the critical flow velocities for the principal bifurcations, as well as nonlinear aspects, such as limitcycle frequencies, amplitudes, and transition between the various dynamical states, are studied in details.

In this paper, the following parameters are used in the simulations: $L=10 \mathrm{~cm}, h=1 \mathrm{~mm}, \rho_{\mathrm{p}}=1200 \mathrm{~kg} \mathrm{~m}^{-3}, \rho_{\mathrm{f}}=$ $1.2 \mathrm{~kg} \mathrm{~m}^{-3}, D=2454 \mathrm{~N} \mathrm{~cm}^{2}$ and $\eta_{\mathrm{d}}=0.0002$. The dimensionless flow velocity $\Pi$ is taken as the bifurcation parameter to explore the system dynamics, while the other parameters remain fixed.

\section{Linear dynamics}

The linear dynamics of the system is examined by obtaining the eigenfrequencies of the linearised version of Eq. (10) around the origin, as the dimensionless flow velocity is varied. The evolution of the generally complex eigenfrequencies of the system as a function of the flow velocity are shown as an Argand diagram in Fig. 2 (top). At low flow velocities, the free motion of the inverted flag is damped. This is reflected in the positive imaginary part of eigenfrequencies. At a sufficiently high flow velocity, the frequency of the first mode becomes purely imaginary, which then bifurcates on the $\Im(\omega)$-axis. One of the solution branches associated with this mode eventually vanishes altogether (i.e. $\Re(\omega)=0, \Im(\omega)=0$ ) at about $\Pi=1.36$ and crosses from 

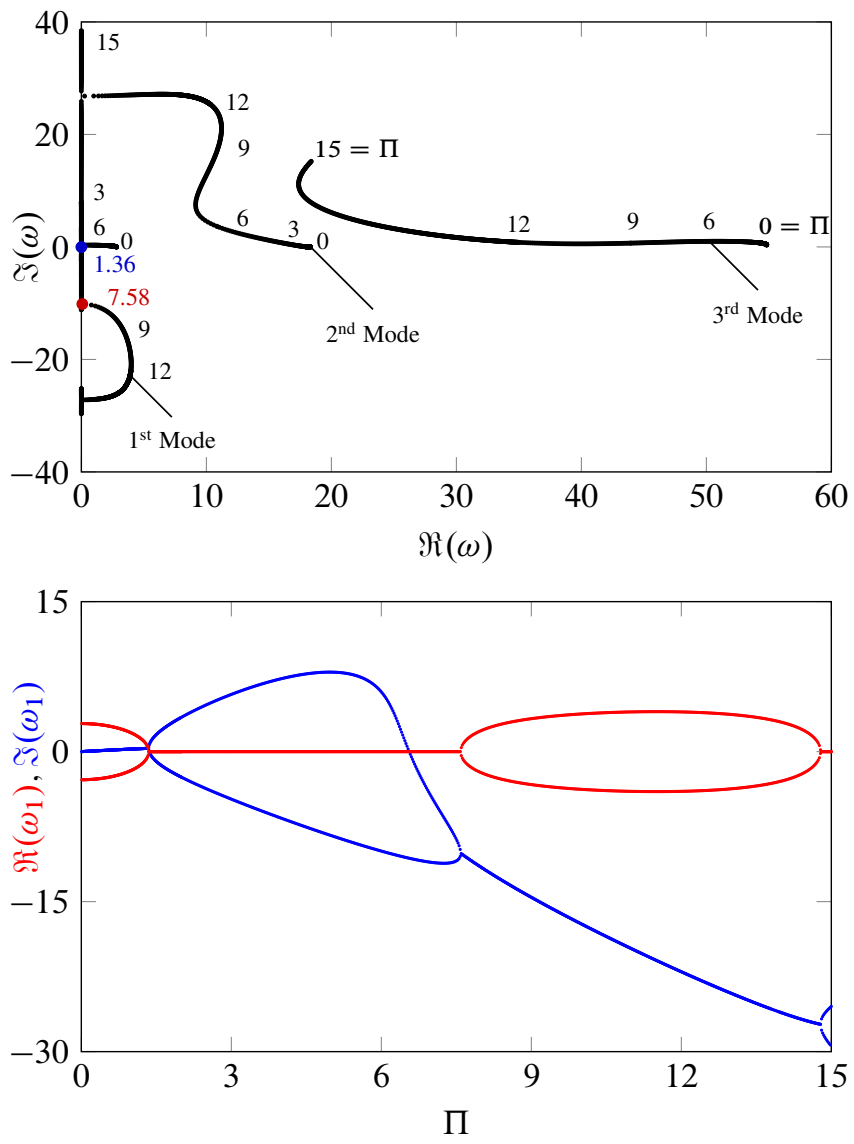

FIGURE 2: (Top) Argand diagram for an inverted flag with $\mu=$ 1.0 showing the evolution of the first three modes as a function of the dimensionless flow velocity $\Pi$. The coupled-mode flutter is of the Païdoussis type. (Bottom) First complex frequency with respect to flow velocity: [-] imaginary part and [-] real part.

the positive to the negative half-plane vertically, indicating the onset of static divergence (buckling). Further increasing the flow velocity gives rise to the coalescence of the negative and positive branches of the first mode loci at $\Pi=7.58$, where the resulting branch leaves the imaginary axis with $\Im(\omega)<0$, indicating the onset of Païdoussis-type coupled-mode flutter [20,21]. Ultimately, the eigenfrequency of the first mode becomes purely imaginary once again at a higher flow velocity $(\Pi=14.74)$, generating a second buckling of the inverted flag in the first mode. More details are shown in Fig. 2 (bottom).

Let us now consider how well linear theory predicts the dynamical behaviour of the system as observed in the experiments. For the physical system, the inverted flag loses its straight, undeformed configuration and buckles similarly to a column under axial load. Thereafter, the flag exhibits flapping motion. By further increasing the flow velocity, the flapping motion gradually diminishes in magnitude, and the flag displays a buckled shape once again but with a larger amplitude of deformation (compared to the initial buckling) as the fluid dynamic forces grow with increasing flow velocity. Thus, up to this point the linear model successfully predicts, qualitatively at least, the dynamics of the physical system. Of course, what concerns the subsequent bifurcations of the system, one cannot expect a linear model to predict, nor what concerns amplitudes of oscillation. To this end, a nonlinear model detailed in the next section is needed.

The stability map shown in Fig. 3 indicates the sensitivity of the foregoing instabilities to the mass ratio. It is noted that the

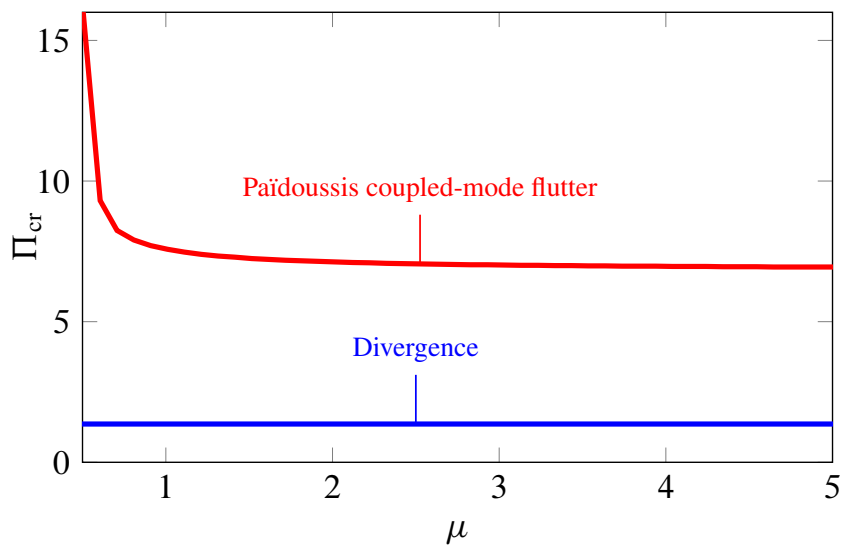

FIGURE 3: Stability map for the investigated 2-D inverted flag: [-] onset of divergence and [-] onset of flutter.

stability of the stretched-straight state of the inverted flag up to the occurrence of divergence is not affected by the mass ratio parameter. Moreover, since the system loses stability by divergence, the mass ratio does not change the value of that critical flow velocity. The onset of flapping, on the other hand, is affected by the mass ratio. More specifically, the Païdoussis-type coupled-mode flutter occurs at considerably higher values of $\Pi$ as $\mu$ is decreased, in the range $\mu \in\left[\begin{array}{ll}0.1 & 0.6\end{array}\right]$. For larger values of $\mu$, the change is less significant, and the curve corresponding to the flutter boundary almost reaches a plateau.

The divergence instability mechanism was originally suggested by Kim et al. [5] through experimental observation, whereby they concluded that the mass ratio does not affect the onset of flapping. A similar mechanism theoretically discovered by Sader et al. [3] via a linear stability analysis. They argued that the divergence instability of the inverted flag is a steady process, where time independent aerodynamic forces are involved. Then, unsteadiness in the flow comes into play and leads to flapping motion. This can be examined further by means of the present nonlinear model.

It should be remarked here that the stability of the original equilibrium predicted by the linear model is valid. However, the post-critical dynamical behaviour may be different from the linear model predictions, once nonlinear effects are taken into account.

\section{Nonlinear dynamics}

In order to investigate the nonlinear aspects of the system dynamics, such as limit-cycle frequencies and amplitudes, as well as the transition between the various dynamical states, the nonlinear integro-partial differential model given in Eq. (10) is solved numerically. The results are plotted in the form of bifurcation diagrams.

The nonlinear response of the two-dimensional flag is shown in Fig. 4. The bifurcation diagram illustrates four distinct regimes, which can be summarized as follows.

Stretched-straight state As expected from linear analysis, the undeflected static equilibrium trivial solution is stable at small 
flow velocities. Small perturbations generate a motion which ultimately dies out, and the inverted flag remains stable.

Buckled state The undeflected static equilibrium loses stability at point $\mathrm{A}$ where $\Pi=1.36$ through a supercritical pitchfork bifurcation. This leads to divergence (buckling) of the first mode, the amplitude of which increases with flow velocity.

Deformed-flapping state The statically deflected solution loses stability via a supercritical Hopf bifurcation at point B where $\Pi=1.70$, leading to a periodic flapping motion around the buckled state. The shape of the response resembles the first mode of a cantilevered beam. The deformed-flapping motion lasts until $\Pi=1.82$, and the amplitude of oscillation increases with $\Pi$ in the range of $\Pi \in\left[\begin{array}{lll}1.70 & 1.82\end{array}\right]$.

Large-amplitude flapping state Increasing the flow velocity further causes a transition from the deformed-flapping regime to flapping around the undeflected trivial equilibrium, the amplitude of which increases with the flow velocity. The transition is accompanied by a jump in the amplitude of flapping via two saddle-node bifurcations at points $\mathrm{C}$ and $\mathrm{D}$ corresponding to $\Pi=1.82$ and $\Pi=1.65$, respectively. This forms a region where the response of the system is attracted by either a stable limit-cycle around the deflected equilibrium or that around the undeflected equilibrium. Hence, the behaviour of the system is indeed subcritical. The existence of this bi-stable zone implies the existence of an unstable repelling limit cycle bounded by the two saddle-node bifurcations.

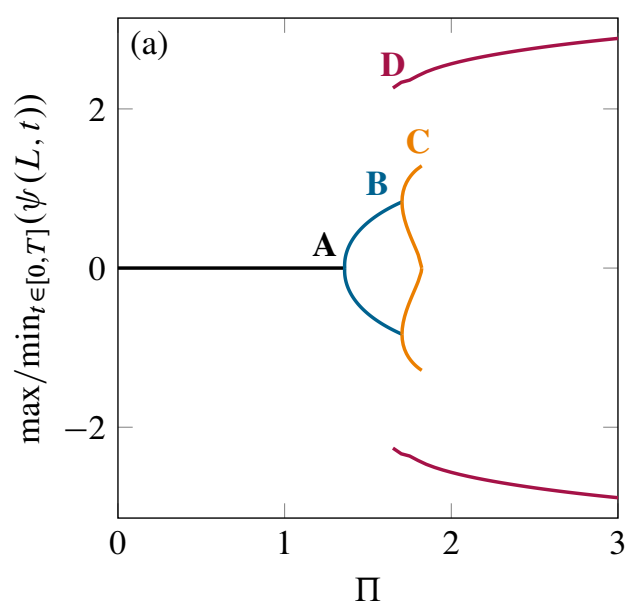

(b)

(c)

(d)

(e)
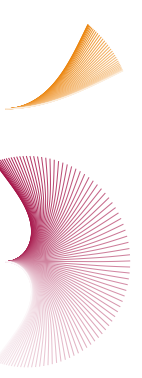

FIGURE 4: (a) Bifurcation diagram for the investigated twodimensional inverted flag with $\mu=1.0$, indicating the peak values of the leading edge rotation over a cycle of steady-state oscillation of period $T^{*}$. [-] Stable static solution (undeflected stable equilibrium); [-] stable static solutions (deflected static equilibria) corresponding to buckling of the inverted flag to either side; [ - ] stable periodic solutions (oscillation around deflected equilibria) corresponding to deformed-flapping of the inverted flag to either side; [-] stable periodic solution (oscillations around undeflected equilibrium). Shapes of the flag corresponding to each regime: (b) stretched-straight state $(\Pi=1.35)$, (c) buckled state $(\Pi=1.40)$, (d) deformed-flapping state ( $\Pi=1.75)$, and (e) large-amplitude flapping $(\Pi=1.65)$.

The model developed in the present study is believed to accurately

simulate the effects of the fluid forces and thus to predict reasonably well the dynamical behaviour of the system up to moderate angles of attack. It is nevertheless realized that the flag exhibits highly curved shapes in the course of large-amplitude flapping (see Fig. 4(e)), signaling that the limit of applicability of inviscid flow theory may have been surpassed. At very large angles of attack, massive separation occurs from the leading edge of the flag, resulting in a sharp drop in the normal force, which in turn causes a sudden decrease in the amplitude of oscillation. This phenomenon is not captured by inviscid flow theory which, in principle, cannot model such viscous effects.

Interestingly, the 'qualitative route' (i.e. stability, buckling, deformed-flapping, and large-amplitude flapping), as well as the critical values of $\Pi$ predicted by the present analytical model, all are in very good agreement with the fully-coupled computational study of Goza et al. [22], who conducted simulations using an immersed boundary method. For example, as shown in Fig. 5, for a two-dimensional inverted flag with $\mu=0.2$ (which is the reciprocal of the dimensionless mass ratio used in [22], there denoted by $M_{\rho}=5.0$ ), the present model predicts the onset of divergence (the pitchfork bifurcation) at $\Pi_{\mathrm{A}}=1.36$, the onset of deformed-flapping (Hopf bifurcation) at $\Pi_{\mathrm{B}}=1.71$, and the onset of large-amplitude flapping (the saddle-node bifurcation) at $\Pi_{\mathrm{D}}=1.80$, while those predicted by the computational model in [22] are $\Pi_{A}=1.42, \Pi_{B}=1.55$, and $\Pi_{D}=1.78$, respectively.

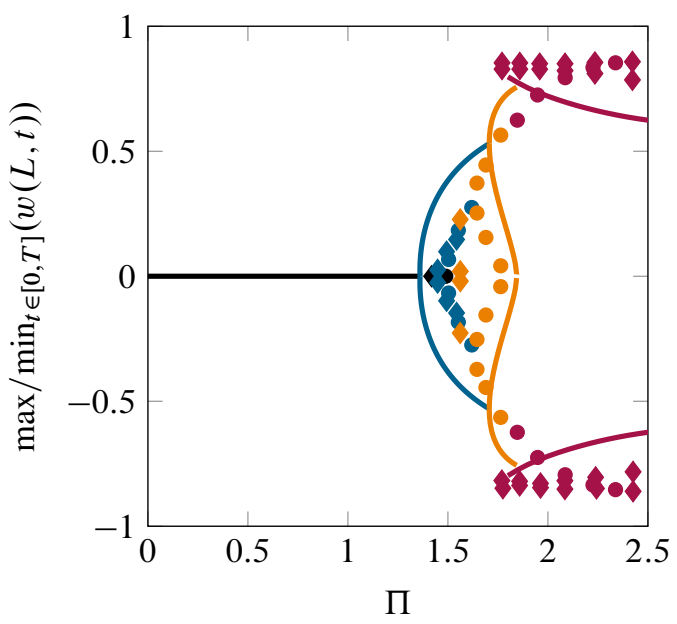

FIGURE 5: Bifurcation diagrams for the investigated inverted flag with $\mu=0.2$, showing the peak values of the tip deflection of the two-dimensional inverted flag over a cycle of steady-state oscillation of period $T^{*}$. [-] stretched-straight state; [-] buckled state; [-] deformed-flapping regime; and [-] large-amplitude flapping around the origin. Lines show the results obtained by the present model, while symbols show the results reported in [22],

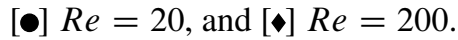

The numerical results discussed here suggest that 'fluid-elastic instabilities' may be the underlying mechanism for such a rich dynamical behaviour displayed by two-dimensional inverted flags. Thus, flapping motions may be viewed as 'self-excited vibration' or flutter. The same mechanism is responsible for flapping of conventional flags in axial flows. However, a number of stud- 
ies on the dynamics of inverted flags suggest the existence of a vortex-induced vibration (VIV) mechanism for flapping [3]. Although several aspects of the dynamics of inverted flags may be explained through the VIV mechanism, the emergence of flapping motion for low mass ratio flags in low Reynolds number flows, i.e. $R e<50$ [22], where vortex shedding does not occur, may pose a challenge to the credence of the VIV mechanism. Further studies ought to be done to provide more insight into the mechanisms governing the dynamics of inverted flags.

\section{Influence of mass ratio}

As discussed in the foregoing, the undeflected equilibrium of the inverted flag loses stability first via divergence, and the post-buckling response of the system remains static prior to the occurrence of a Hopf bifurcation. As seen from Eq. (10), the mass ratio parameter $\mu$ is present only in the time-dependent terms. Thus, it is expected that $\mu$ should have no impact on the critical point for divergence or the existence of the deflected equilibria. This conclusion agrees well with experimental/theoretical findings of Sader et al. [3] for the onset of divergence. The existence of a deflected equilibrium following the onset of divergence was realised in their experiments by introducing additional damping by touching the flag with a slender rigid pole.

The mass ratio $\mu$, on the other hand, does affect the oscillatory dynamical behaviour of the system and has a significant impact on the stability of the associated solution branches. Figure 6 depicts the effect of $\mu$ on the nonlinear response of the system. It is
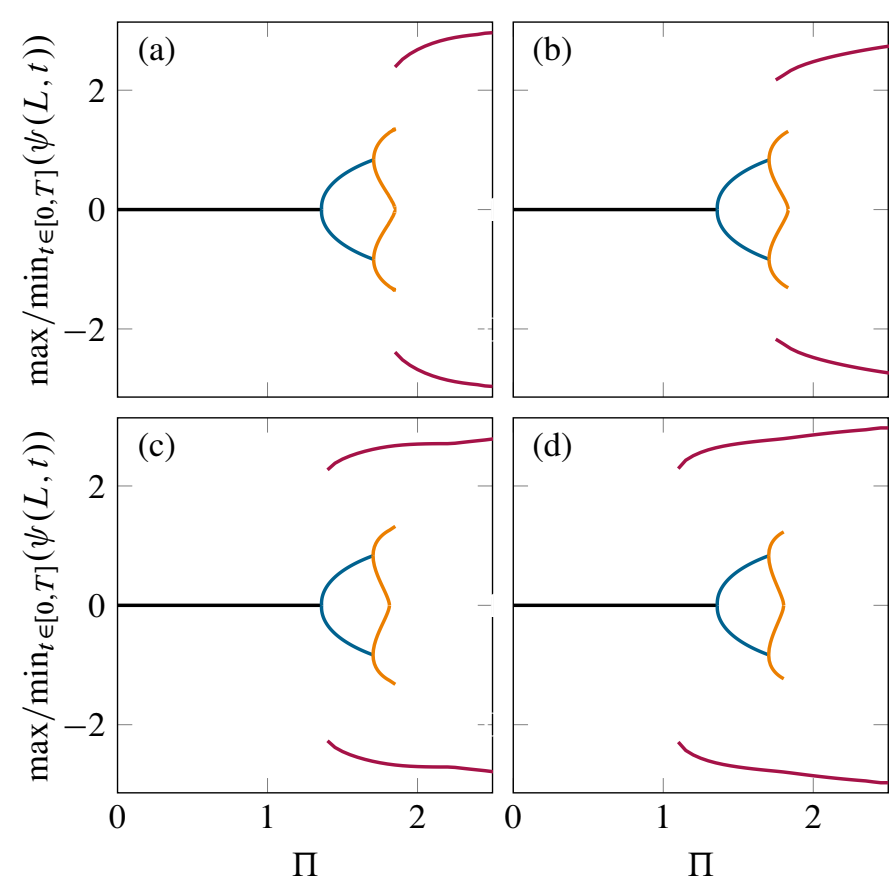

FIGURE 6: Bifurcation diagrams for a two-dimensional inverted flag with various mass ratios: (a) $\mu=0.1$, (b) $\mu=0.5$, (c) $\mu=2.0$ and (d) $\mu=5.0$, indicating the peak values of the leading edge rotation angle over a cycle of steady-state oscillation of period $T^{*}$. The colour scheme in figure 4 is used.

seen that the stability of the trivial solution, and the critical flow velocities for divergence and virtually the Hopf bifurcation, are not affected by $\mu$. The post-critical behaviour, on the other hand, is significantly altered. For instance, for the case with $\mu=0.5$, the critical values of the flow velocity are $\Pi_{\mathrm{A}}=1.36, \Pi_{\mathrm{B}}=1.70$, $\Pi_{\mathrm{C}}=1.83$, and $\Pi_{\mathrm{D}}=1.75$, while for the case with $\mu=5.0$ these values are $\Pi_{\mathrm{A}}=1.36, \Pi_{\mathrm{B}}=1.70, \Pi_{\mathrm{C}}=1.80$, and $\Pi_{\mathrm{D}}=$ 1.40 .

For sufficiently large $\mu$ (i.e. for a light flag or a heavilyloaded flag), the transition to large-amplitude flapping becomes strongly subcritical. A stable periodic solution around the position of rest originates form point $\Pi_{D}$ at a flow velocity even lower than that for the onset of divergence. The existence of this solution would give rise to the possibility of a direct jump from static undeflected equilibrium to large-amplitude flapping motion, without encountering other states. More specifically, considering the case with $\mu=5.0$ as shown in Fig. 6(d), two stable solutions coexist for $\Pi \in[1.101 .36]$ : the trivial solution and a large-amplitude periodic solution. Considering $\Pi=1.20$, for instance, small perturbations about the undeflected equilibrium die out, and the inverted flag returns to the original static equilibrium. Perturbations of sufficiently large amplitude, on the other hand, could lead the system to undergo large-amplitude flapping, without experiencing the usual route outlined earlier.

It is noted that the critical flow velocity for the Hopf bifurcation $\Pi_{B}$ obtained via the nonlinear model is considerably lower than that obtained via the linearised model. This may be explained by considering the fact that a linear model assumes that an instability emanates from the position of rest where the deflections are vanishingly small. Once divergence develops, however, the amplitude increases with flow velocity. As the deflection becomes larger, the nonlinear fluid-related forces become dominant. This generates a Hopf bifurcation, leading to a limit cycle oscillation, to occur at considerably different, and in this case lower, flow velocities, compared to the predictions by the linear model.

The findings of the present paper are based on several assumptions, such as that the flag lies within a two-dimensional quasi-steady flow. These assumptions might limit the capability of the model to accurately predict the dynamics of the flag, for example, when very a large flapping motion occurs. Nevertheless, the findings of this paper motivate further research on the analytical modelling of inverted flags subject to axial flow. Such modelling can provide deep insights into the underlying mechanisms for the various instabilities, and the impact of different parameters on the global as well as local dynamics and stability of the system.

\section{CONCLUSION}

In the present paper, it was shown that the mechanism for flapping of infinitely high aspect ratio inverted flags in axial flow may be through 'fluid-elastic instability'. In other words, flapping of two-dimensional inverted flags may be viewed as a 'self-excited (or movement-induced) vibration' similarly to flutter of an aircraft wing or flutter of a cantilevered pipe conveying fluid.

The numerical results showed that two-dimensional inverted flags undergo multiple bifurcations as the flow velocity is increased. Physically-speaking, the flags exhibit four dynamical states (or regimes) with increasing the flow velocity: (i) stretched-straight state, (ii) buckled state, (iii) deflected-flapping state, and (iv) largeamplitude flapping state. 
It was shown that the system dynamics depends on the mass ratio parameter. The mass ratio does not affect the stability of the stretched-straight state and the onset of divergence, as they are static phenomena; however, it controls the possibility of direct transition from static undeflected equilibrium to large-amplitude flapping motion, and it affects the amplitude of large-amplitude flapping. In addition, inverted flags of larger mass ratios are more prone to undergo flapping motion at lower flow velocities due to the presence of a subcritical periodic solution. At a certain critical flow velocity (corresponding to a saddle-node bifurcation), flowinduced disturbances may result in spontaneous large-amplitude flapping of flags with sufficiently large mass ratio.

The outcome of this study may be of practical importance for the design of small-scale energy harvesting systems. The proposed analytical model is believed to be a promising tool for analyzing some aspects of such systems, with reasonable computational burden, which can be employed in order to perform sensitivity analysis, or be utilized in optimization schemes and control algorithms.

\section{ACKNOWLEDGMENT}

The authors gratefully acknowledge the financial support offered by the Natural Sciences and Engineering Research Council of Canada, the Solution Mining Research Institute (SMRI) and the Pipeline Research Council International (PRCI). The last author also appreciates the financial support by Concordia University through a Start-Up grant.

\section{REFERENCES}

[1] Guo, C. Q., and Païdoussis, M., 2000. "Stability of rectangular plates with free side-edges in two-dimensional inviscid channel flow". Journal of Applied Mechanics, 67(1), pp. 171176. doi:10.1115/1.321143.

[2] Orrego, S., Shoele, K., Ruas, A., Doran, K., Caggiano, B., Mittal, R., and Kang, S. H., 2017. "Harvesting ambient wind energy with an inverted piezoelectric flag". Applied Energy, 194, pp. 212-222. doi:10.1016/j.apenergy.2017.03.016.

[3] Sader, J., Cossé, J., Kim, D., Fan, B., and Gharib, M., 2016. "Large-amplitude flapping of an inverted flag in a uniform steady flow-a vortex-induced vibration". Journal of Fluid Mechanics, 793, pp. 524-555. doi:10.1017/jfm.2016.139.

[4] Fan, B., Huertas-Cerdeira, C., Cossé, J., Sader, J., and Gharib, M., 2018. Effect of morphology on the large-amplitude flapping dynamics of an inverted flag in a uniform flow. arXiv: 1810.13308 .

[5] Kim, D., Cossé, J., Huertas Cerdeira, C., and Gharib, M., 2013. "Flapping dynamics of an inverted flag". Journal of Fluid Mechanics, 736. doi:10.1017/jfm.2013.555.

[6] Kornecki, A., Dowell, E., and O'Brien, J., 1976. "On the aeroelastic instability of two-dimensional panels in uniform incompressible flow". Journal of Sound and Vibration, 47(2), pp. 163-178. doi:10.1016/0022-460X(76)90715-X.

[7] Sader, J., Huertas-Cerdeira, C., and Gharib, M., 2016. "Stability of slender inverted flags and rods in uniform steady flow". Journal of Fluid Mechanics, 809, pp. 873-894. doi:10.1017/jfm.2016.691.

[8] Tavallaeinejad, M., Legrand, M., and Païdoussis, M., 2018. "Nonlinear response of inverted flags subjected to a steady flow". In 9th International Symposium on Fluid-Structure Interactions, Flow-Sound Interactions, Flow-Induced Vibration \& Noise. July 8-11, Toronto, Canada, hal-01812870.

[9] Tavallaeinejad, M., Païdoussis, M., and Legrand, M., 2018. "Nonlinear static response of low-aspect-ratio inverted flags subjected to a steady flow". Journal of Fluids and Structures, 83, pp. 413-428. hal-01745147.

[10] Theodorsen, T., 1949. General theory of aerodynamic instability and the mechanism of flutter. Tech. rep., NASA. hdl-19930090935.

[11] Polhamus, E., 1966. A concept of the vortex lift of sharpedge delta wings based on a leading-edge-suction analogy. Tech. rep., NASA. 19670003842.

[12] Bisplinghoff, R., Ashley, H., and Halfman, R., 2013. Aeroelasticity. Courier Corporation.

[13] Ramesh, K., Gopalarathnam, A., Edwards, J., Ol, M., and Granlund, K., 2013. "An unsteady airfoil theory applied to pitching motions validated against experiment and computation". Theoretical and Computational Fluid Dynamics, 27(6), pp. 843-864. doi:10.1007/s00162-012-0292-8.

[14] Yan, Z., Taha, H., and Hajj, M., 2014. "Geometricallyexact unsteady model for airfoils undergoing large amplitude maneuvers". Aerospace Science and Technology, 39, pp. 293306. doi:10.1016/j.ast.2014.09.021.

[15] Katz, J., and Weihs, D., 1981. "Wake rollup and the Kutta condition for airfoils oscillating at high frequency". AIAA Journal, 19(12), pp. 1604-1606.

[16] Katz, J., and Plotkin, A., 2001. Low-Speed Aerodynamics, Vol. 13. Cambridge University Press.

[17] Crighton, D. G., 1985. "The Kutta condition in unsteady flow". Annual Review of Fluid Mechanics, 17(1), pp. 411445.

[18] Hodges, D., and Pierce, A., 2011. Introduction to Structural Dynamics and Aeroelasticity, second ed. Cambridge University Press.

[19] Gear, C. W., 1971. "The automatic integration of ordinary differential equations". Communications of the ACM, 14(3), pp. 176-179. doi:10.1145/362566.362571.

[20] Done, G., and Simpson, A., 1977. "Dynamic instability of certain conservative and non-conservative systems". Journal of Mechanical Engineering Science, 19(6), pp. 251-263. doi:10.1243/JMES_JOUR_1977_019_053_02.

[21] Païdoussis, M., 2014. Fluid-Structure Interactions: Slender Structures and Axial Flow, second ed., Vol. 1. Oxford Academic Press.

[22] Goza, A., Colonius, T., and Sader, J., 2018. "Global modes and nonlinear analysis of inverted-flag flapping". Journal of Fluid Mechanics, 857, pp. 312-344. doi:10.1017/jfm.2018.728. 